\title{
Percutaneous Balloon Pericardiotomy by the Use of Inoue Balloon for the Management of Recurrent Cardiac Tamponade in a Patient with Lung Cancer
}

\author{
Masashi OhKe, Akihiro Bessho, Kayo Haraoka, Tsuyoshi Ohgou, Shinji Ozaki and Takumi Kishimoto
}

\begin{abstract}
A 32-year-old man with lung cancer involving pericarditis carcinomatosa underwent pericardiotomy, using an Inoue balloon dilating catheter, to create a non-surgical pericardial window. The procedure was performed from the thoracic wall to the left pleural effusion and parietal pericardium under local anesthesia. The effects of non-surgical pericardial window had been maintained until this patient died from his primary disease. It is concluded that percutaneous balloon pericardiotomy is helpful in the management of massive pericardial effusions particularly in patients with malignancies and poor clinical condition. (Internal Medicine 39: 1071-1074, 2000)
\end{abstract}

Key words: pericardial effusion, pericarditis carcinomatosa, pericardiocentesis, non-surgical pericardial window

\section{Introduction}

Malignancy is one of the common causes of pericardial effusion with tamponade, tachyarrhythmias, or congestive heart failure. The incidence of metastatic malignancies to the myocardium and the pericardium has been described from less than $1 \%$ to more than $20 \%$ of all cancer patients and this incidence varies with the type of malignancy (1-4). The management of pericardial effusion in patients with malignancy is an important problem that confronts both the oncologist and the cardiologist. The prognosis of patients with malignant cardiac tamponade is related to early diagnosis and treatment of tamponade. A common problem is that malignant pericardial effusions reaccumulate rapidly and call for more radical treatment. The recommended therapy for recurrent malignant pericarditis has been described to be either multiple pericardiocentesis accompanied by instillation of chemotherapeutic drugs or the cre- ation of a subxiphoid surgical window (5-7). Surgical evacuation of pericardial effusion under pressure can be accomplished with various techniques (8-11). Such techniques are performed under general anesthesia. However a 30-day mortality rate has been reported (9) with this procedure in up to $60 \%$ of documented cases. This probably reflects the severity of the systemic illness. For this reason, surgery should be avoided if possible. In 1991, Palacios et al (12) first described percutaneous balloon pericardiotomy and demonstrated its effectiveness and relatively low risk. Later, Chow and Chow (13) reported the use of the Inoue balloon catheter for this procedure. The technique of percutaneous balloon pericardiotomy is less invasive than surgery and has more constant positive effects than pericardiocentesis. We describe our experience of percutaneous balloon pericardiotomy using the Inoue balloon in the management of malignant pericardial effusion induced by lung cancer.

\section{Case Report}

A 32-year-old man with non-small cell (adenocarcinoma) lung cancer was admitted to our hospital with malignant pericardial effusion (Fig. 1). An urgent pericardiocentesis through the subxiphoid approach was performed. Following removal of $1,020 \mathrm{ml}$ of bloody pericardial fluid, cisplatin was administrated into the pericardium to prevent a recurrence of pericardial effusion. The cytology of the pericardial effusion revealed adenocarcinoma. Subsequently, he received systemic chemotherapy consisting of 2 cycles of a combination of cisplatin 80 $\mathrm{mg} / \mathrm{m}^{2}$, once daily i.v. on day 1 and etoposide $100 \mathrm{mg} / \mathrm{m}^{2}$, once daily i.v. on days 1,2 , and 3 , but the size of the main tumor of the lung became enlarged. Six months later, the patient was admitted again with cardiac tamponade due to reaccumulation of pericardial effusion. Echocardiography confirmed the development of moderate pericardial effusion only behind his heart with left ventricular collapse (Fig. 2). An urgent pericardiocentesis was performed through the left pleural effusion approach. Following removal of $600 \mathrm{ml}$ of bloody peri-

From the Department of Internal Medicine, Okayama Rousai Hospital, Okayama

Received for publication December 10, 1999; Accepted for publication July 6, 2000

Reprint requests should be addressed to Dr. Masashi Ohke, the Department of Internal Medicine, Okayama Rousai Hospital, 1-10-25 Chikkoumidorimachi, Okayama 702-8055 


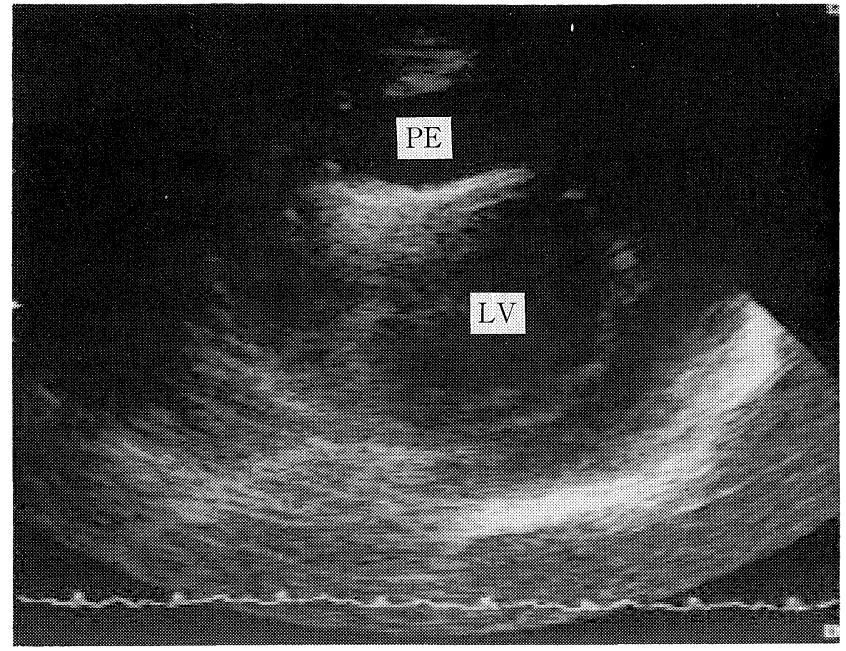

Figure 1. Echocardiography confirmed large pericardial effusion. PE: pericardial effusion, LV: left ventricle.

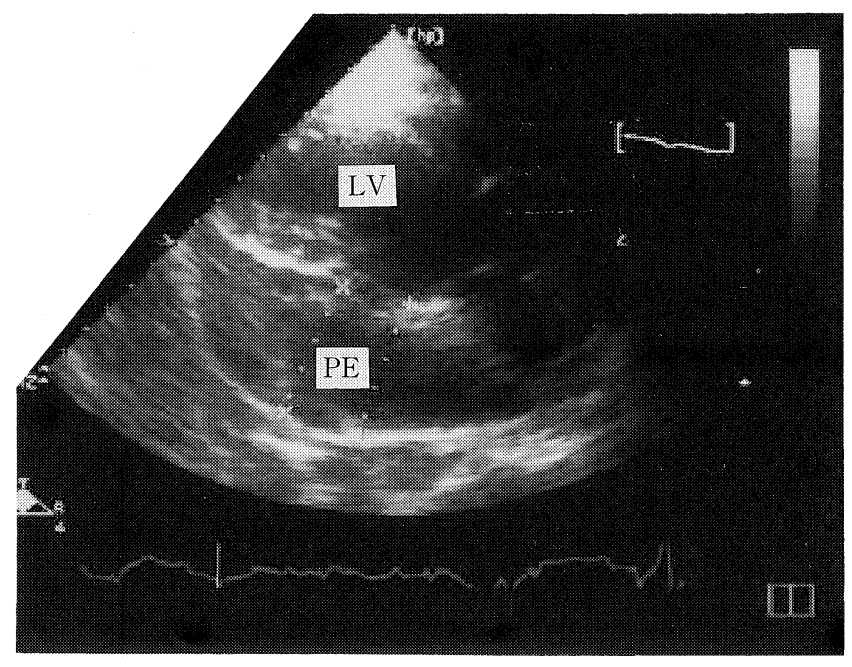

Figure 2. Echocardiography confirmed the development of moderate pericardial effusion behind the heart with left ventricular collapse. PE: pericardial effusion, $L V$ : left ventricle.

cardial fluid, continuous drainage could be achieved by placing a pigtail catheter in the pericardial space, but the effusion was not controllable. To avoid a new development of cardiac tamponade, we decided to create a pericardial window by employing the percutaneous balloon pericardiotomy method described by Chow and Chow (13). In brief, after administration of local anesthesia a 0.025 inch guide-wire was inserted into the pericardial space from the left chest wall through the pigtail catheter. A 12 french dilator was advanced over the guidewire, through the thoracic wall, left pleural effusion and parietal pericardium. A self-positioning catheter, the Inoue balloon catheter set (Toray Medical Co. Ltd., Tokyo, Japan) at $22 \mathrm{~mm}$,

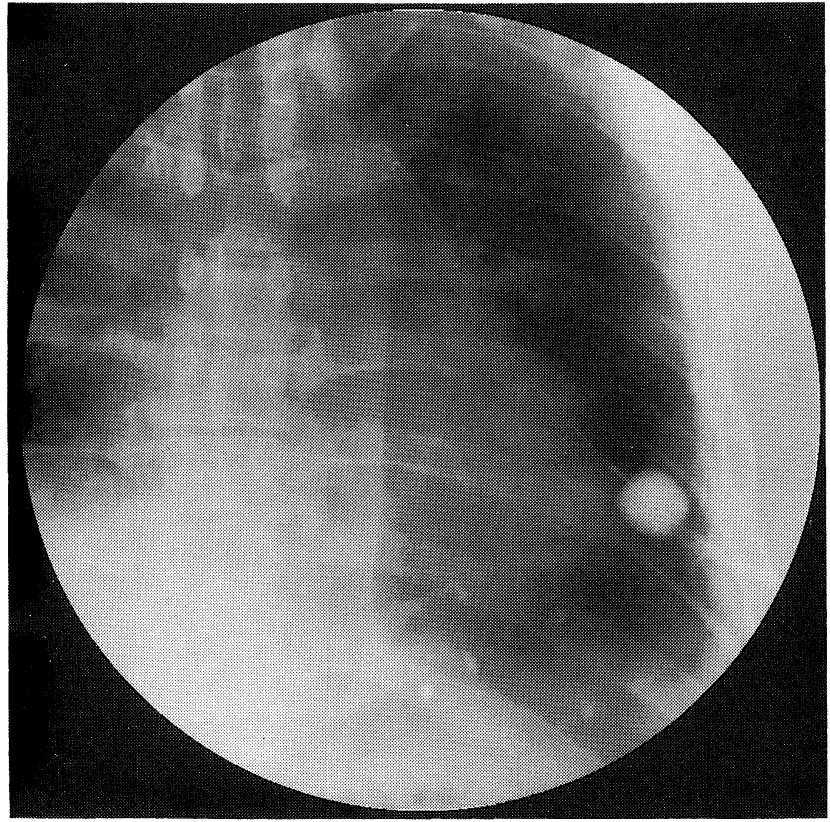

Figure 3. The distal balloon anchored itself at the parietal pericardium.

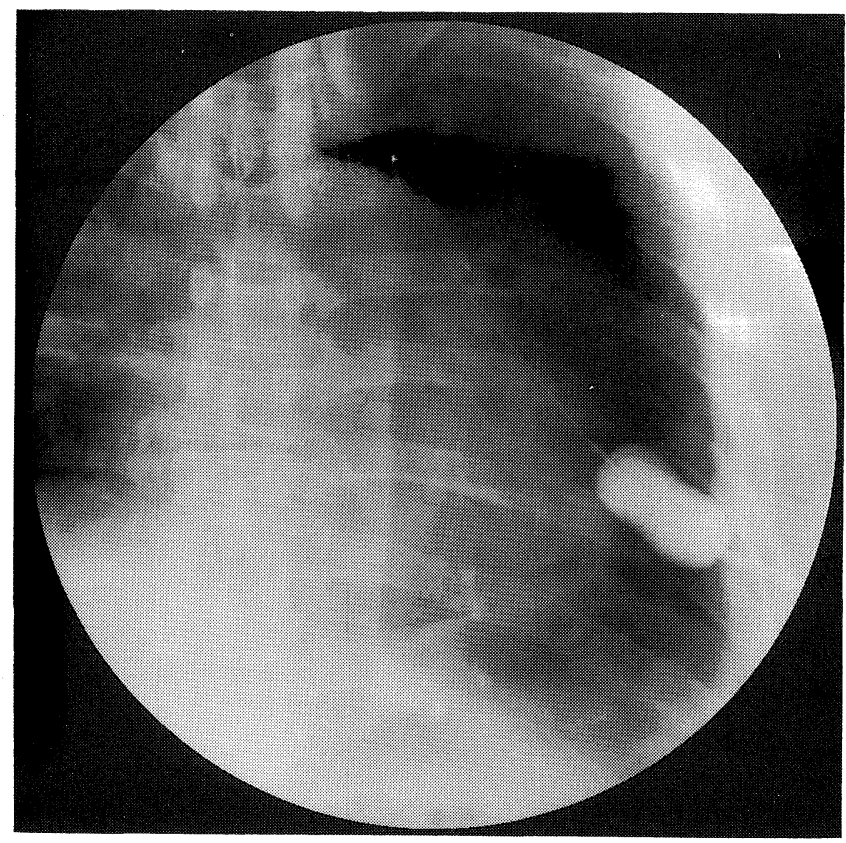

Figure 4. The balloon catheter was then rapidly inflated until the waist disappeared.

was exchanged over the guide-wire and advanced into the pericardial space under fluoroscopy. The distal portion of the balloon was inflated first and the catheter was then pulled back gently until the distal balloon anchored itself at the parietal pericardium (Fig. 3). The balloon was then rapidly inflated to 
its full extent until the waist disappeared (Fig. 4). It was kept inflated for 1 minute. Three inflations were performed to ensure adequate opening of the pericardium. After this procedure, we recognized that contrast medium was washed out from the pericardial space to the pleural cavity. Follow-up on the patient was performed via echocardiograph for seven months after this procedure. No signs of reaccumulation of the malignant pericardial effusion were observed. He died of respiratory failure due to lung cancer.

\section{Discussion}

Recently, balloon pericardiotomy has been proposed as a useful and less invasive therapy which is an alternative to surgical pericardiotomy (14-18). This technique can be performed even in critically ill patients with malignant pericardial tamponade, with a high rate of procedural success. Ziskind et al (19) already reported the effectiveness of creating a pericardial window with percutaneous balloon pericardiotomy and described a success rate of $93 \%$ with no severe complications except for 3\% displaying fever and $1 \%$ showing pneumothorax. In Europe and America, several authors have reported the usefulness of this procedure, whereas there have been no such case reports compiled in Japan. The frequency of the recurrent pericardial effusion with pericarditis carcinomatosa is comparatively little. However, the recurrent pericardial effusion is often localized, and occasionally recurs over 6 months after the first drainage. Here, we performed pericardiotomy by using the Inoue balloon to treat recurrent pericardial effusion induced by lung cancer. During the inflating of the balloon, no clinical changes, including heart rates and blood pressure, were observed. As mentioned in many previous reports, the procedure is simple and safe and could be performed under local anesthesia and mild sedation. However, in Japan the indication of this procedure cannot be the first choice, due to the high cost of the Inoue balloon. We suggested that the indication of balloon pericardiotomy should be restricted to cases of cardiac tamponade suffering recurrence of localized pericardial effusion in spite of extensive efforts of drainage by using a pigtail catheter or cases not allowing further insertion of drainage catheter at the next recurrence of effusion. Although our patient had recurrent pericardial effusions localized posterior to his heart, we could insert a pigtail catheter without injuring the lung because of the presence of pleural effusion. But it proved difficult to do a second insertion. Therefore, we performed the pericardiotomy by using the Inoue balloon. The technique is relatively simple, safe, and can be performed under local anesthesia and mild sedation, with minimal discomfort even in critically ill patients. Previous reports $(14-16,18)$ described that the balloon should be inflated two to three times. For this procedure, we performed three inflations to ensure an adequate opening of the pericardium. Confirmation established that contrast medium was washed out from the pericardial space to the pleural cavity. To data, the efficacy of balloon pericardiotomy versus pericardiocentesis as a primary treatment for malignant pericardial effusion is still unknown. But it may become the pre- ferred treatment to avoid more invasive procedures for patients with a limited life expectancy.

\section{Conclusion}

We have presented a case of recurrent cardiac tamponade treated with percutaneous balloon pericardiotomy by the use of the Inoue balloon. The patient reaccumulated fluid following pericardiocentesis, but we achieved good long-term results with balloon pericardiotomy. During the seven months of follow-up, his pericardial effusion did not significantly reaccumulate. Percutaneous balloon pericardiotomy helps to manage pericardial effusion and improve the quality of life. The procedure is relatively simple, safe and can be performed under local anesthesia and mild sedation.

\section{References}

1) Kapoor AS. Clinical manifestations of neoplasia of the heart. Cancer and the Heart. Springer-Verlag, New York, 1986: 21-25.

2) Kline IK. Cardiac lymphatic involvement by metastatic tumor. Cancer 29: 799-808, 1972.

3) Lockwood WB, Broghamer WL Jr. The changing prevalence of secondary cardiac neoplasms as related to cancer therapy. Cancer 45: 26592662, 1980.

4) Roberts WC, Bodey GP, Wertlake PT. The heart in acute leukemia: a study of 420 autopsy cases. Am J Cardiol 21: 388-412, 1968.

5) Davis S, Sharma SM, Blumberg ED, Kim CS. Intrapericardial tetracycline for the management of cardiac tamponade secondary to malignant pericardial effusion. N Engl J Med 299: 1113-1114, 1978.

6) Flannery EP, Gregoratos G, Corder MP. Pericardial effusionsin patients with malignant disease. Arch Intern Med 135: 976-977, 1975.

7) Shepherd FA, Morgan C, Evans WK, Ginsberg JF, Watt D, Murphy K. Medical management of pericardial effusion by tetracycline sclerosis. Am J Cardiol 60: 1161-1166, 1987.

8) Miller JI, Mansour KA, Hatcher CR Jr. Pericardiotomy: Current indication, concepts, and results in university center. Ann Thorac Surg 45: 26592662, 1980.

9) Palatianos GM, Thurer RJ, Kaiser GA. Comparison of effectiveness and safety of operations on the pericardium. Chest 88: 30-33, 1985.

10) Park JS, Rentschler R, Wilbur D. Surgical management of pericardial effusion in patients with malignancies. Cancer 67: 76-80, 1991.

11) Piehler JM, Pluth JR, Schaff HV, Danielson GK, Orszulak TA, Fuga FJ. Surgical management of effusive pericardial disease. J Thorac Cardiovasc Surg 90: 506-516, 1985.

12) Palacios IF, Tuzcu EM, Ziskind AA, Younger J, Block PC. Percutaneous balloon pericardial window for patients with malignant pericardial effusion and tamponade. Cathet Cardiovasc Diagn 22: 244-249, 1991.

13) Chow LT, Chow WH. Mechanism of pericardial window creation by balloon pericardiotomy. Am J Cardiol 72: 1321-1322, 1993.

14) Chow WH, Chow TC, Yip AS, Cheung KL. Inoue balloon pericardiotomy for Patients with Recurrent Pericardial Effusion. Angiology 47: 57-60, 1996.

15) Thanopoulos BD, Georgakopoulos DG, Tsaousis GS, Triposkiadis F, Paphitis CA. Percutaneous balloon pericardiotomy for the treatment of large, nonmalignant pericardial effusions in children: immediate and Medium-Term Results. Cathet Cardiovasc Diagn 40: 97-100, 1997.

16) Hsu KL, Tsai CH, Chiang FT, et al. Percutaneous balloon pericardiotomy for Patients With Recurrent Pericardial Effusion: Using a Novel DoubleBalloon Technique With One Long and One Short Balloon. Am J Cardiol 80: 1635-1637, 1997.

17) Law DA, Haque R, Jain A. Percutaneous balloon pericardiotomy: nonsurgical treatment for patients with cardiac tamponade. W V Med J 93: 


\section{OHKE et al}

310-312, 1997.

18) Galli M, Politi A, Jemoli R, Zerboni S. Transcatheter management of cardiac and pericardial metastatic involvement. G ltal Cardiol 28: 687$690,1998$.
19) Ziskind AA, Pearce AG, Lemmon CC, et al. Percutaneous balloon pericardiotomy for the treatment of cardiac tamponade and large pericardial effusions: Description of technique and report of the first 50 cases. J Am Coll Cardiol 21: 1-5, 1993. 